\title{
GPS and the Search for Axions
}

\section{A. Nicolaidis}

Theoretical Physics Department, Aristotle University of Thessaloniki, Thessaloniki, Greece

Email: nicolaid@auth.gr

How to cite this paper: Nicolaidis, A. (2017) GPS and the Search for Axions. Journal of Modern Physics, 8, 1470-1477. https://doi.org/10.4236/jmp.2017.88089

Received: May 15, 2017

Accepted: July 21, 2017

Published: July 24, 2017

Copyright $\odot 2017$ by author and Scientific Research Publishing Inc. This work is licensed under the Creative Commons Attribution International License (CC BY 4.0).

http://creativecommons.org/licenses/by/4.0/

\begin{abstract}
GPS, an excellent tool for geodesy, may serve also particle physics. In the presence of Earth's magnetic field, a GPS photon may be transformed into an axion. The proposed experimental setup involves the transmission of a GPS signal from a satellite to another satellite, both in low orbit around the Earth. To increase the accuracy of the experiment, we evaluate the influence of Earth's gravitational field on the whole quantum phenomenon. There is a significant advantage in our proposal. While the geomagnetic field $B$ is low, the magnetized length $L$ is very large, resulting into a scale $(B L)^{2}$ orders of magnitude higher than existing or proposed reaches. The transformation of the GPS photons into axion particles will result in a dimming of the photons and even to a "light shining through the Earth" phenomenon.
\end{abstract}

\section{Keywords}

Axions, Earth Magnetic Field, Quantum Mechanics, Earth’s Gravity, Artificial Satellites

\section{Introduction}

Quantum Chromodynamics (QCD) describes the strong interactions among quarks and gluons and offers definite predictions at the high energy-perturbative domain. At low energies, the non-linear nature of the theory introduces a nontrivial vacuum which violates the $\mathrm{CP}$ symmetry. The $\mathrm{CP}$ violating term is parameterized by $\theta$ and experimental bounds indicate that $\theta \leq 10^{-10}$. The smallness of $\theta$ is known as the strong $\mathrm{CP}$ problem.

An elegant solution has been offered by Peccei-Quinn [1] [2] [3]. A global $\mathrm{U}(1)_{\mathrm{PQ}}$ symmetry is introduced, the spontaneous breaking of which provides the cancellation of the $\theta$-term. As a byproduct, we obtain the axion field, the NambuGoldstone boson of the broken $\mathrm{U}(1)_{\mathrm{PQ}}$ symmetry. There are extensive reviews covering the theoretical aspects and the experimental searches for the axion [4]-[9]. 
A general feature of the axion is its two-photon coupling

$$
L_{a_{\gamma \gamma}}=-\frac{1}{4} g a F_{\mu \nu} \tilde{F}^{\mu v}=g a \boldsymbol{E} \cdot \boldsymbol{B}
$$

where $\alpha$ is the axion field, $F_{\mu v}\left(\tilde{F}^{\mu v}\right)$ the (dual) electromagnetic field strength tensor and $g$ the photon-axion coupling constant. Accordingly, in the presence of a magnetic field $\boldsymbol{B}$, a photon may oscillate into an axion and vice-versa. A prototype experiment in the search for solar axions is CAST experiment, which set the limit for $g<10^{-10} \mathrm{GeV}^{-1}$ [10]. The CAST experiment involves a magnetic field $B=9 \mathrm{~T}$ and a magnetized region $L=9.3 \mathrm{~m}$. Therefore, the relevant scale $(B L)^{2}$ is $(B L)^{2} \approx 7000 \mathrm{~T}^{2} \mathrm{~m}^{2}$. Always in the search for solar axions, a space-based experiment has been proposed, where use is made of the Earth's magnetic field [11]. The weakness of the geomagnetic field $B$ is somehow compensated by the larger $L$ value.

In the present work, we suggest using the geomagnetic field in order to study the inverse process, photon-axion transition. We consider a GPS signal travelling from one satellite to another. In the presence of the Earth's magnetic field, the photon may oscillate to an axion and vice-versa. Our proposal involves an increased $(B L)^{2}$ scale, a higher accuracy and the exploration of a new range of $g$, $m_{a}$ (coupling constant and axion mass respectively). To further increase the accuracy of our evaluation, we include the effect of Earth's gravitational field on the whole process.

\section{GPS Signal and the Influence of Earth's Gravitational Field}

Global Positioning System (GPS) offers to geodesy position measurements with a millimeter to centimeter-level precision. GPS contributed also to significant advances in geophysics, seismology, atmospheric science and natural hazard science. Besides accurate positioning, all disturbances in the propagation of the transmitted GPS signal from satellite to receiver are mined for information [12]. The GPS system is in effect a realization of Einstein's view of space and time. Indeed, the system cannot function properly without taking into account fundamental relativistic principles [13].

In the presence of the geomagnetic field we may envisage the transition of the GPS signal into an axion. To reach the highest accuracy in the evaluation of the probability $P(\gamma \rightarrow \alpha)$, we must include also the effect of Earth's gravitational field.

The geometry outside a spherical star like the Earth is provided by the Schwarzschild metric

$$
\mathrm{d} \tau^{2}=\left(1-\frac{R_{\mathrm{s}}}{r}\right) \mathrm{d} t^{2}-\left(1-\frac{R_{\mathrm{s}}}{r}\right)^{-1} \mathrm{~d} r^{2}-r^{2}\left(\mathrm{~d} \theta^{2}+\sin ^{2} \theta \mathrm{d} \phi^{2}\right)
$$

where $R_{s}=2 G M$ with $M$ the mass of the star. The relevant scale in our problem, with $R_{g}$ the radius of the GPS satellite from the Earth's center, is

$$
\frac{R_{s}}{R_{g}}=\frac{2 G M_{E}}{R_{g}} \approx 3 \times 10^{-10}
$$


Given the smallness of the gravitational strength, we adopt from the very start the metric of a weak gravitational field

$$
\mathrm{d} \tau^{2}=\left(1-\frac{R_{s}}{r}\right) \mathrm{d} t^{2}-\left(1+\frac{R_{s}}{r}\right)\left[\mathrm{d} x^{2}+\mathrm{d} y^{2}+\mathrm{d} z^{2}\right]
$$

The metric is independent of the coordinate $t$ and this implies that the energy is conserved

$$
m\left(1-\frac{R_{s}}{r}\right) \frac{\mathrm{d} t}{\mathrm{~d} \tau}=p_{0} \equiv E=\text { const }
$$

The relation

$$
g^{\mu v} p_{\mu} p_{v}=m^{2}
$$

provides

$$
\frac{E^{2}}{\left(1-R_{s} / r\right)}-\frac{p^{2}}{\left(1+R_{s} / r\right)}=m^{2}
$$

where we defined $p^{2}=\boldsymbol{p}^{2}=\sum_{i=1}^{3} p_{i}^{2}$.

The quantum mechanical phase accumulated by a particle propagated in space-time is given by the invariant quantity [14]-[19]

$$
\mathrm{d} \Phi=p_{\mu} \mathrm{d} x^{\mu}=E \mathrm{~d} t-\sum_{i} p_{i} \mathrm{~d} x^{i}
$$

Using the relation

$$
p_{i}=m\left(1+\frac{R_{s}}{r}\right) \frac{\mathrm{d} x^{i}}{\mathrm{~d} \tau}
$$

and the relation (5), we obtain

$$
p_{i}=E \frac{\left(1+R_{s} / r\right)}{\left(1-R_{s} / r\right)} \frac{\mathrm{d} x^{i}}{\mathrm{~d} t}
$$

Subsequently,

$$
p=E \frac{\left(1+R_{s} / r\right)}{\left(1-R_{s} / r\right)} \frac{\mathrm{d} s}{\mathrm{~d} t}
$$

where $\mathrm{ds} s^{2}=\mathrm{d} x^{2}+\mathrm{d} y^{2}+\mathrm{dz}^{2}$.

The quantum phase acquires the form

$$
\mathrm{d} \Phi=E \mathrm{~d} t-p \mathrm{~d} s=\left(E \frac{\mathrm{d} t}{\mathrm{~d} s}-p\right) \mathrm{d} s=\left[E^{2} \frac{\left(1+R_{s} / r\right)}{\left(1-R_{s} / r\right)}-p^{2}\right] \frac{\mathrm{d} s}{p}
$$

Equation (7) allows to rewrite

$$
\mathrm{d} \Phi=m^{2} \frac{\left(1+R_{s} / r\right)}{p} \mathrm{~d} s
$$

The above expression is accurate within the weak gravity approach. Notice that in the absence of gravity we obtain

$$
\mathrm{d} \Phi_{0}=\frac{m^{2}}{p} \mathrm{~d} s
$$


which is the well-known established result for the Minkowski spacetime.

Working always in the weak gravity limit and ignoring terms $\left(R_{s} / r\right)^{2}$, we evaluate, using Equation (7)

$$
\frac{\left(1+R_{s} / r\right)}{p} \approx \frac{1}{\left[E^{2}-\frac{m^{2}}{\left(1+R_{s} / r\right)}\right]^{1 / 2}} \approx \frac{1}{\sqrt{E^{2}-m^{2}}}-\frac{1}{2} \frac{R_{s}}{r} \frac{m^{2}}{\left(E^{2}-m^{2}\right)^{3 / 2}}
$$

We conclude that

$$
\mathrm{d} \Phi=\frac{m^{2}}{\sqrt{E^{2}-m^{2}}}\left[1-\frac{1}{2} \frac{R_{s}}{r} \frac{m^{2}}{\left(E^{2}-m^{2}\right)}\right] \mathrm{d} s
$$

In our case we consider a light signal travelling from a satellite at $r=R_{g}$ to another satellite at $r=R_{g}$.

The trajectory is almost a straight line and the closest distance to the Earth is denoted by $b$. Then the traveled distance is $s=2 \sqrt{R_{g}^{2}-b^{2}}$ and

$$
\Phi_{0}=\frac{m^{2}}{\sqrt{E^{2}-m^{2}}} 2 \sqrt{R_{g}^{2}-b^{2}}
$$

For the second contribution we have to evaluate the integral $\int \frac{\mathrm{d} s}{r}$.

Defining $\cos \phi=\frac{b}{r}$ we obtain

$$
\int \frac{\mathrm{d} s}{r}=\int \frac{\mathrm{d} \phi}{\cos \phi}
$$

where $0 \leq \phi \leq \phi_{\max }$ with $\cos \phi_{\max }=\frac{b}{R_{g}}$.

We obtain finally

$$
\Phi=\frac{m^{2}}{\sqrt{E^{2}-m^{2}}}\left[2 \sqrt{R_{g}^{2}-b^{2}}-\frac{m^{2}}{E^{2}-m^{2}} R_{s} \ln \frac{(1+\omega)}{(1-\omega)}\right]
$$

with

$$
\omega=\left[\frac{R_{g}-b}{R_{g}+b}\right]^{1 / 2}
$$

It should be noted that the energy $E$ is the energy measured at infinity $r=\infty$. The energy $E$ and the energy $E_{g}$ measured at distance $r=R_{g}$ (the position of the satellites) are connected by

$$
E=E_{g}\left(1-R_{s} / R_{g}\right)^{1 / 2}
$$

The above relation represents the well-known gravitational red shift. Expressing everything in terms of the measured $E_{g}$ and considering the case $m^{2} \ll E^{2}$ we find the compact expression

$$
\Phi=\frac{m^{2}}{E_{g}}\left(1+\frac{R_{s}}{2 R_{g}}\right)\left(2 \sqrt{R_{g}^{2}-b^{2}}\right)
$$


We conclude that the influence of the Earth's gravitational field can be absorbed into a definition of an effective mass $\mu$

$$
\mu^{2}=m^{2}\left(1+\frac{R_{s}}{2 R_{g}}\right)
$$

\section{Photon-Axion Oscillations}

Consider a GPS signal travelling from a satellite at $r=R_{g}$ to another satellite at $r$ $=R_{g}$, both moving at low altitude around the Earth. Let us define as $z$ axis the direction of photon's propagation. The polarization of the photon $\boldsymbol{A}$ lies then at the $\mathrm{x}-\mathrm{y}$ plane. The photon is moving in the presence of the geomagnetic field $\boldsymbol{B}$. The component of $\boldsymbol{B}$ parallel to the direction of motion does not induce photon-axion mixing. Following Equation (1), the transverse magnetic field $\boldsymbol{B}_{\mathrm{T}}$ couples to $A_{\| l}$, the photon polarization parallel to $\boldsymbol{B}_{\mathrm{T}}$ and decouples from $\mathrm{A}_{\perp}$, the photon polarization orthogonal to $\boldsymbol{B}_{\mathrm{T}}$.

The photon-axion mixing is governed by the following equation:

$$
\left(E_{g}-i \vartheta_{z}+\mathbf{M}\right)\left(\begin{array}{c}
A_{1 \mid} \\
a
\end{array}\right)=0
$$

The 2-dimensional matrix $\mathbf{M}$ is

$$
\mathbf{M}=\left(\begin{array}{cc}
-\frac{\mu_{\gamma}^{2}}{2 E_{g}} & \frac{g B_{\mathrm{T}}}{2} \\
\frac{g B_{\mathrm{T}}}{2} & -\frac{\mu_{a}^{2}}{2 E_{g}}
\end{array}\right)
$$

where $\mu^{2}$ is defined in Equation (22). For a photon, moving in a medium with number density of electrons $n_{e}$, the photon mass $m_{\gamma}$ is given by

$$
m_{\gamma}^{2}=\frac{4 \pi \alpha n_{e}}{m_{e}}
$$

The axion mass $m_{\alpha}$ is not experimentally known. Matrix $\mathbf{M}$ is diagonalized through the angle $\theta$ with

$$
\tan 2 \theta=\frac{2 g B_{\mathrm{T}} E_{g}}{\mu_{a}^{2}-\mu_{\gamma}^{2}}
$$

Defining

$$
\begin{gathered}
D=\frac{1}{2 E_{g}}\left[\left(\mu_{a}^{2}-\mu_{\gamma}^{2}\right)^{2}+4 g^{2} B_{\mathrm{T}}^{2} E_{g}^{2}\right]^{1 / 2} \\
\sin 2 \theta=\frac{g B_{\mathrm{T}}}{D}
\end{gathered}
$$

we obtain for the probability that a photon converts into an axion after travelling a distance $s$

$$
P(\gamma \rightarrow \alpha)=\sin ^{2} 2 \theta \sin ^{2} \frac{D s}{2}
$$


When the oscillatory term in Equation (29) is small, i.e. $D s / 2 \ll 1$, we obtain the behavior $(L \equiv s)$

$$
P_{0}(\gamma \rightarrow \alpha)=\frac{g^{2}}{4}\left(B_{\mathrm{T}} L\right)^{2}
$$

thus the relevant scale for an experimental setup is $\left(B_{\mathrm{T}} L\right)^{2}$.

Imagine a photon scratching the Earth at $b=R_{E}$ and becoming an axion at this position.

The probability is

$$
P(\gamma \rightarrow \alpha)=\sin ^{2} 2 \theta \sin ^{2}\left(\frac{D}{2} \sqrt{R_{g}^{2}-R_{E}^{2}}\right)
$$

The axion reemerging from the Earth travels to the other satellite. The probability of being detected there like a photon is

$$
P(\alpha \rightarrow \gamma)=\sin ^{2} 2 \theta \sin ^{2}\left(\frac{D}{2} \sqrt{R_{g}^{2}-R_{E}^{2}}\right)
$$

Therefore the probability for light shining through the Earth is given by

$$
P=\sin ^{4} 2 \theta \sin ^{4}\left(\frac{D}{2} \sqrt{R_{g}^{2}-R_{E}^{2}}\right)
$$

\section{Discussion and Overall Conclusions}

We suggest a new experimental technique, where thanks to the Earth's magnetic field, a GPS signal is transformed to an axion particle. There are clear advantages in our proposal.

First, the high accuracy. The distance $L$ is measured with a precision at the millimeter-centimeter level. Second, the large value of the $(B L)^{2}$ scale. The Earth's magnetic field is of a dipole form with a mean value $B_{0} \approx 3 \times 10^{-5} \mathrm{~T}$ on the Earth's surface. The magnetic field is falling off like $\left(R_{E} / r\right)^{3}$, where $R_{E}$ is the radius of the Earth (approximately $6370 \mathrm{~km}$ ) and $r$ the radial distance from the center of the Earth. To obtain the best available values for the geomagnetic field $\left(\approx B_{0}\right)$, the satellites should remain in a low orbit around the Earth. Actually, rather than working with present GPS, we should use inexpensive microsatellites, known as cubesats, orbiting close to the Earth's surface. The smallness of the magnetic field, compared say, to the CAST experiment, is overbalanced by the much larger $L$ value, which may reach $L=2 R_{E}$. The scale $(B L)^{2}$ becomes then $140,000 \mathrm{~T}^{2} \mathrm{~m}^{2}$, orders of magnitude above existing or proposed values. Third, the energy range of the photons and the possibility to search in an unexplored domain of $g, m_{\alpha}$ values. GPS photons travel with a frequency of approximately 1 GHz. It is appropriate, for our experimental needs, to use a higher frequency of 1 $\mathrm{THz}$, so that we can reach lower values for $m_{\alpha}$, down to $\mu \mathrm{eV}$ [20] and explore even smaller $g$ values. Finally, our emitter and receiver are in constant motion and therefore the parameter $L$ may vary offering plentiful information. Relying on Einstein's view of space and time, GPS has been established as the ideal tool for geodesy. Next to the relativistic conceptions we included a quantum approach, and we obtained the probability for the transition of a GPS photon to an 
axion particle in the presence of the Earth's magnetic field. This transition will result in a dimming of the photons and further to light shining through the Earth phenomenon. We may envisage that in the future the long list of scientific disciplines served by GPS will be enriched by particle physics.

\section{Acknowledgements}

The present work was initiated while I was a visiting scholar at the Center for Axion and Precision Physics, Institute for Basic Science (CAPP-IBS) in Korea. I would like to thank CAPP's director Prof. Yannis Semertzidis for the kind invitation and many enlightening discussions. Aspects of GPS related technology were clarified during a visit at the Laboratoire Astroparticule et Cosmologie (APC) in Paris. I am appreciative of this help provided by APC's director Prof. Stavros Katsanevas. Mr. Dimitris Evangelinos assisted in the typesetting.

\section{Note Added}

Our proposal involves the combination of relativity theory and quantum theory, in order to carry out a quantum particle experiment in space. One might question the prevalence of quantum principles in light propagation in outer space over a long distance. But the recently announced, after our paper appeared, Chinese achievement, with the Micius satellite sending entangled photons over thousands of kilometers [21], proves the feasibility of the proposal.

\section{References}

[1] Peccei, R.D. and Quinn, H.R. (1977) Physical Review Letters, 38, 1440. https://doi.org/10.1103/PhysRevLett.38.1440

[2] Weinberg, S. (1978) Physical Review Letters, 40, 223. https://doi.org/10.1103/PhysRevLett.40.223

[3] Wilczek, F. (1978) Physical Review Letters, 40, 279. https://doi.org/10.1103/PhysRevLett.40.279

[4] Raffelt, G. and Stodolsky, L. (1988) Physical Review D, 37, 1237. https://doi.org/10.1103/PhysRevD.37.1237

[5] Raffelt, G. (1999) Nuclear Physics B-Proceedings Supplements, 77, 456-461. https://doi.org/10.1016/S0920-5632(99)00467-3

[6] Sikivie, P. (2005) AIP Conference Proceedings, 805, 23 https://doi.org/10.1063/1.2149671

[7] Kim, J. (2016) Talk Given at the Corfu Summer Institute, arXiv:1703.03114[hep-ph].

[8] Graham, P., Irastorza, I., Lamoreaux, S., Lindner, A. and van Bibber, K. (2015) Annual Review of Nuclear and Particle Science, 65, 485-514. https://doi.org/10.1146/annurev-nucl-102014-022120

[9] Battesti, R., Beltran, B., Davoudiasl, H., Kuster, M., Pugnat, P., Rabadan, R., Ringwald, A., Spooner, N. and Zioutas, K. (2008) Lecture Notes in Physics, 741, 199-237. https://doi.org/10.1007/978-3-540-73518-2_10

[10] Zioutas, K., et al. (CAST Collaboration) (2005) Physical Review Letters, 94, 121301. https://doi.org/10.1103/PhysRevLett.94.121301

[11] Davoudiasl, H. and Huber, P. (2006) Physical Review Letters, 97, 141302. 
https://doi.org/10.1103/PhysRevLett.97.141302

[12] Bock, Y. and Melgar, D. (2016) Reports on Progress in Physics, 79, 106801. https://doi.org/10.1088/0034-4885/79/10/106801

[13] Ashby, N. (2002) Physics Today, 55, 41-72. https://doi.org/10.1063/1.1485583

[14] Stodolsky, L. (1979) General Relativity and Gravitation, 11, 391-405. https://doi.org/10.1007/BF00759302

[15] Fornengo, N., Giunti, C., Kim, C.W. and Song, J. (1977) Physical Review D, 56, 1895-1902. https://doi.org/10.1103/PhysRevD.56.1895

[16] Pereira, J.G. and Zhang, C.M. (2000) General Relativity and Gravitation, 32, 1633 1637. https://doi.org/10.1023/A:1001902706237

[17] Ahluwalia, D.V. and Burgard, C. (1996) General Relativity and Gravitation, 28, 1161-1170. https://doi.org/10.1007/BF03218936

[18] Ahluwalia, D.V. and Burgard, C. (1998) Physical Review D, 57, 4724. https://doi.org/10.1103/PhysRevD.57.4724

[19] Bhattacharya, T., Habib, S. and Mottola, E. (1999) Physical Review D, 59, 067301. https://doi.org/10.1103/PhysRevD.59.067301

[20] Borsyanyi, S., et al. (2016) Nature, 539, 69-171. https://doi.org/10.1038/nature20115

[21] Yin, J., et al. (2017) Science, 356, 1140-1144. https://doi.org/10.1126/science.aan3211

Submit or recommend next manuscript to SCIRP and we will provide best service for you:

Accepting pre-submission inquiries through Email, Facebook, LinkedIn, Twitter, etc. A wide selection of journals (inclusive of 9 subjects, more than 200 journals) Providing 24-hour high-quality service User-friendly online submission system Fair and swift peer-review system Efficient typesetting and proofreading procedure Display of the result of downloads and visits, as well as the number of cited articles Maximum dissemination of your research work

Submit your manuscript at: http://papersubmission.scirp.org/

Or contact jmp@scirp.org 